\title{
Patrimoine urbain, lieux de mémoire et fonctions de capitale à Berlin, de l'Unité allemande à la réunification
}

Urban heritage, places of remembrance and capital city functions in Berlin from the German Unity to the Reunification

Städtisches Erbe, Erinerrungsorte und Haupstadtfunktionen in Berlin (EinheitWende)

\section{Géraldine Djament-Tran}

\section{OpenEdition}

\section{Journals}

Electronic version

URL: http://journals.openedition.org/rge/5034

DOI: $10.4000 /$ rge.5034

ISSN: 2108-6478

\section{Publisher}

Association des géographes de l'Est

\section{Printed version}

Date of publication: 30 December 2013

ISSN: 0035-3213

\section{Electronic reference}

Géraldine Djament-Tran, «Patrimoine urbain, lieux de mémoire et fonctions de capitale à Berlin, de I'Unité allemande à la réunification », Revue Géographique de l'Est [Online], vol. 53 / 3-4 | 2013, Online since 02 July 2014, connection on 08 September 2020. URL : http://journals.openedition.org/rge/5034 ; DOI : https://doi.org/10.4000/rge.5034

This text was automatically generated on 8 September 2020

Tous droits réservés 


\title{
Patrimoine urbain, lieux de mémoire et fonctions de capitale à Berlin, de l'Unité allemande à la réunification
}

\author{
Urban heritage, places of remembrance and capital city functions in Berlin from \\ the German Unity to the Reunification \\ Städtisches Erbe, Erinerrungsorte und Haupstadtfunktionen in Berlin (Einheit- \\ Wende)
}

Géraldine Djament-Tran

Au sein du paradigme national-patrimonial (Bobbio, 1992), le patrimoine et les capitales entretiennent un rapport structurel et dialectique, abordé ici dans une optique constructiviste $^{1}$. Le patrimoine, ensemble des biens jugés dignes d'être transmis à la postérité, construit comme composante maitresse de l'iconographie nationale, légitime les fonctions de capitale. Réciproquement, le statut de capitale, siège du pouvoir politique, induit un processus de patrimonialisation, par lequel un groupe social extrait un objet de la vie sociale normale pour lui accorder le statut d'emblème de son identité dans le temps (Micoud, 2005), qui à son tour participe de la production d'une communauté imaginée nationale (Anderson, 1996). Erigé en appareil idéologique d'Etat (Althusser, 1976), le patrimoine participe aux fonctions politiques - les lieux de pouvoir sont fréquemment des lieux patrimonialisés -, aux fonctions de représentation et aux fonctions culturelles de la capitale, vitrine, emblème et creuset supposé de la nation.

Le cas de Berlin se révèle un observatoire particulièrement riche de cette coconstruction du patrimoine et de la capitale au service du nation making process. En effet, la dialectique classique du patrimoine et de la capitale s'y trouve complexifiée par de fréquents changements de statut politique, qui induisent des cycles rapprochés de redéfinition conjointe des lieux de pouvoirs de la capitale et de ce qui fait patrimoine dans la capitale ${ }^{2}$. Le statut de capitale de Berlin varie en effet tant dans son contenu 
territorial (la capitale de la Prusse devient en 1871 capitale de l'Allemagne unifiée ; les frontières allemandes sont fortement modifiées en 1945, la ligne Oder-Neisse sanctionnant la perte territoriale à l'est) que politique. Capitale du Deuxième Reich, de la République de Weimar puis du Troisième Reich, elle devient ensuite pour sa moitié est capitale de la RDA, tandis que sa moitié ouest perd son statut de capitale au profit de Bonn ; la ville entière perd le statut de capitale au moment de la réunification, avant de devenir, à la suite du vote du 20 juin 1991, et effectivement en 2000, à la suite du déménagement des organes fédéraux, capitale de l'Allemagne réunifiée. La place géographique de Berlin évolue parallèlement: capitale politique, mais aussi, notamment après la Première Guerre Mondiale, économique et culturelle, elle voit avec la guerre froide sa centralité décliner au profit d'un système urbain polycentrique. Ces vicissitudes historiques ont posé successivement la question d'une capitale et d'un patrimoine post-nazis puis post-socialistes, occasionnant des choix indissociablement politiques et urbanistiques: faut-il détruire, réutiliser et/ou patrimonialiser les vestiges des régimes renversés?

En outre, le schéma de renforcement mutuel unissant le patrimoine aux fonctions de capitale dysfonctionne à Berlin à partir de la seconde moitié du XXe siècle. En effet, deux événements majeurs ont perturbé le système national-patrimonial classiquement mis en place à partir de la fin du XIXe siècle : le nazisme et la coupure entre RDA et RFA. Le premier, héritage encombrant par excellence, soulevant la question du patrimoine négatif (Wahnich, 2011), a fait peser sur l'idée de nation allemande et donc sur la capitale et le patrimoine de la nation, l'hypothèque d'un nationalisme exacerbé. La seconde a remis en cause l'existence d'une seule communauté imaginée allemande : depuis 1991, la capitale et son patrimoine ne connaissent pas un « simple » changement de régime, mais une réunification, qui pose la question du retour (pour la capitale comme pour le patrimoine) à l'état de référence antérieur à la coupure du rideau de fer. Ce Sonderweg ${ }^{3}$ conduit Berlin à hésiter depuis la réunification entre un patrimoine post-national, dans la lignée des options ouest-allemandes de la guerre froide (François, 1992), et un patrimoine que l'on pourrait qualifier de néo-national ${ }^{4}$.

\section{LE PARADIgME NATIONAL-PATRIMONIAL DANS LA CAPITALE ALLEMANDE : MISE EN PLACE ET PERTURBATIONS}

En Allemagne, dès la fin du XVIIIe siècle, "les processus de nation-builing et de memory-building se déterminent et se renforcent réciproquement» (François, 2001, p. 13).

\section{A. La mise en place du paradigme national-patrimonial dans le cadre de la constitution des fonctions de capitale nationale}

La fixation de sentiments patriotiques sur un objet «patrimonial» peut symboliquement remonter à Berlin à la perte du quadrige de Schadow surmontant la Porte de Brandenburg, emporté par Napoléon en 1806 et réinstallé en 1814 (Seibt in François, Schulze, 2001). 
Le lien entre construction d'une capitale prussienne puis allemande et patrimonialisation se manifeste ensuite notamment dans l'île des Musées, dont le projet remonte à 1797 et prend forme en 1816. Celle-ci complète, avec l'université Humboldt fondée en 1810, les fonctions culturelles de la capitale, en réalisant le projet de musée universel typique des Lumières. Elle contribue également à la production de l'espace urbain de la capitale: en 1822 débute le projet de Schinkel pour l'Altes Museum, conçu comme un temple du Beau et inauguré en 1829, dans le cadre d'une opération d'urbanisme qui remodèle un site capital de Berlin (Poulot, 2001). Schinkel voulait donner à voir à des fins d'éducation populaire les trois piliers de l'Etat: le musée, le château et la cathédrale baroque. La poursuite des constructions sur l'ile des musées jusqu'au IIIe Reich inclus s'inscrit «dans la volonté de rivaliser avec les capitales européennes plus précocément engagées dans la compétition muséale » (ibid., p. 84). Ce complexe patrimonial donne à voir la nation allemande. En particulier, la Nationalgalerie, bâtie à partir de 1866 par Friedrich August Stüler dans un style monumental et inaugurée en 1876 le jour de l'anniversaire de Guillaume Ier, porte à son entrée, précédée par une statue équestre du roi Guillaume IV, l'inscription 'Der Deutschen Kunst MDCCCLXXI', et un fronton consacré à «Germania protectrice des Beaux-Arts ». Des peintures y glorifient les batailles menant à l'Unité allemande. Son second directeur, Hugo von Tschudi, suscitera la polémique par sa politique d'achat de tableaux impressionnistes français, jugés heurter le sentiment national (Aronsson, Bentz, 2011). En 1904 est inauguré le Kaiser-Friedrich Museum, au nom significatif. L'île des musées, dont la protection patrimoniale est assurée par les législations de 1907, 1909 et 1923, articule à l'horizon d'attente national un champ d'expérience reconstruit. Elle représente, avec Munich et le Germanisches Nationalmuseum de Nuremberg, un maillon essentiel de la constitution du système allemand des musées nationaux (Aronsson, Bentz, art. cit.).

La construction de Berlin comme capitale nationale s'accompagne également d'une phase de monumentalisation. Ainsi, le Reichstag conçu en style wilhelmien par Paul Wallot, est inauguré en 1884 (Roeck in François, Schulze, 2001). L'Eglise du Souvenir, construite entre 1890 et 1895 sur les plans de Franz Schwechten, est consacrée au premier empereur d'Allemagne, Guillaume I, et à la victoire de Sedan sur l'armée française en 1870. La Neue Wache, édifiée en 1818 par Schinkel sur Unter den Linden pour abriter la garde du commandement de la garnison de Berlin, devient sous la République de Weimar monument aux morts de la Première Guerre Mondiale.

Berlin connaît en outre une protection précoce de son patrimoine typique des pays germaniques, qui voient apparaître au milieu des années 1890 un mouvement de défense des paysages urbains menacés par l'industrialisation, promoteur de la notion de Stadtbild ${ }^{5}$. Une circulaire de 1904 du ministre prussien de la Religion et de l'Education entraîne une protection patrimoniale large d'un point de vue typologique et chronologique (Poulot, 2001).

La République de Weimar poursuit la construction d'une capitale et d'un patrimoine nationaux, désormais dissociée du projet impérial, lançant un projet avorté de nouveau quartier des ministères et achevant en 1930 la construction du musée de Pergame, qui glorifie « l'appropriation des anciennes civilisations » (Poulot, 2001, p. 85). 


\section{B. Les perturbations du paradigme national-patrimonial à Berlin : I'héritage encombrant du nazisme et le dédoublement patrimonial de la guerre froide}

Ce schéma national-patrimonial se trouve remis en question à la suite du nazisme. Non seulement les destructions de la fin de la guerre endommagent le patrimoine urbain (l'Ile des musées est ainsi bombardée) et posent la question des ruines traumatiques. La ruine violente de l'église du Souvenir, bombardée en novembre 1943, est ainsi conservée, à la suite d'une mobilisation des Berlinois contre le projet de démolition, comme marqueur de l'histoire urbaine, lieu de commémoration et mode de prévention du risque ${ }^{6}$ (Le Blanc, 2010) et intégrée au projet d'Egon Eiermann comprenant une nouvelle église et une grande tour, réalisé au début des années 1960.

Mais surtout, après le nazisme et son nationalisme exacerbé, l'idée de nation allemande entre dans l'ère du soupçon. Se posent alors les questions de la dissociation entre patrimoine et patrie, et de la gestion de l'héritage urbain nazi, particulièrement encombrant. Ainsi la Neue Wache avait été reconvertie en sanctuaire à la gloire des héros nazis. Le régime de RDA choisit de conserver le monument et d'en inverser les symboles, le transformant en mémorial aux victimes du fascisme et du militarisme. Le nazisme transforme également en héritage encombrant les vestiges du militarisme prussien, considéré comme son précurseur. C'est ainsi que la RDA dynamite en 1950 le château des Hohenzollern, pour reconstruire dans les années 1970 le palais de la République, ou renomme en 1956 le Kaiser-Friedrich Museum musée Bode, du nom de son directeur au début du XXe siècle, " pour effacer le nom des Hohenzollern » (Poulot, 2001, p. 85). De même, à l'ouest, le Reichstag fait l'objet à la fin des années 1950 d'une «épuration » du style wilhelmien, avant que Paul Baumgarten ne remporte en 1960 le concours pour une restauration prudente (Roeck, art. cit.). Le bâtiment Martin-Gropius accueille en 1981, à proximité immédiate du mur de Berlin, l'exposition "Preußen.

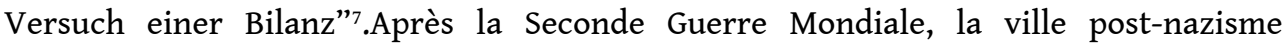
redéfinit ainsi ce qui fait patrimoine au détriment de l'héritage prussien : il n'y a pas retour à l'état de référence immédiatement antérieur au régime encombrant, mais réévaluation globale de l'histoire nationale et méfiance inédite vis-à-vis de la notion même de patrimoine national.

Cependant, la capitale est-allemande pour les fonctions politico-administratives comme la vitrine berlinoise du régime ouest-allemand ayant perdu le statut de capitale au profit de Bonn réemploient des bâtiments appartenant au "patrimoine encombrant " (Dorel-Ferré, 2011) de la ville, en enlevant les symboles politiques nazis les plus explicites (Reichel, 1998). La RDA transforme ainsi le Ministère de l'armée de l'air de l'époque nazie en Maison des Ministères abritant le Ministère des Finances ainsi que tous les ministères compétents pour la planification économique (Laporte, 2012).

Toutefois, le processus le plus marquant de la guerre froide, durant laquelle Berlin devient Frontstadt (la «ville-front »), puis, après 1961, Mauerstadt (la «ville-mur »), réside dans le passage d'un à deux patrimoines berlinois, cherchant à construire deux communautés imaginées allemandes. Les institutions patrimoniales se dédoublent Berlin, compte tenu de sa fonction de vitrine dans l'affrontement des deux régimes, se voit dotée de deux musées de villes ou de deux galeries nationales, conformément au phénomène de "Gegengründungen" (Aronsson, Bentz, art. cit., p. 352) - tandis que les récits et les dispositifs patrimoniaux divergent radicalement. La culture politique 
ouest-allemande, européenne et occidentale, marquée par une dénazification inachevée, le tabou du nazisme et un "rapport absent à l'histoire " contesté par les étudiants en 1968, peut être qualifiée de "post-nationale". Elle produit avec le Musée historique allemand lancé en 1987 comme pendant de la maison de la RFA à Bonn par le chancelier Helmut Kohl ${ }^{9}$ sur un projet de 1982 ayant occasionné des débats coïncidant avec l'Historikerstreit ${ }^{10}$, « un musée post-national ». Il se trouve en effet fondé sur la conviction selon laquelle «l'histoire allemande ne peut trouver son sens (...) que dans un triple dépassement: dans un dépassement européen (...), dans un dépassement chronologique (...) ; dans un dépassement vers l'intérieur " car il existe plusieurs identités allemandes (François, 1992, p. 74). Cette réalisation s'inscrit dans la lignée de l'exposition «Fragen an die deutsche Geschichte " ${ }^{11}$ tenue en 1971 dans l'ancien Reichstag et conçue comme une réponse didactique, présentant les valeurs de la démocratie libérale, au musée de l'histoire allemande de la RDA.

Cet instrument de propagande installé au cœur du Berlin historique, situé symboliquement à proximité du siège du comité central et des principaux ministères dans l'ancien arsenal du roi de Prusse, incarnait quant à lui la vision de l'histoire de la RDA (ibid.). La réécriture marxiste-stalinienne de l'histoire urbaine s'y manifeste également dans les changements toponymiques ou la destruction du château des Hohenzollern, sur l'emplacement duquel est construit le palais de la République, cœur politique et culturel du régime. Toutefois, la RDA ne fait pas du passé urbain nécessairement table rase, mais recherche une légitimité historique en reconstruisant partiellement l'avenue Unter den Linden ou, pour les 750 ans de Berlin en 1987, le quartier Saint Nicolas, et en restaurant la porte de Brandenbourg (Buffet, 1999).

La période 1933-1989 correspond ainsi à une configuration spécifiquement berlinoise, qui passe de l'association paradigme national-patrimonial hypertrophiéfonctions de capitale totalitaire au dédoublement entre l'association paradigme patrimonial post-national-perte des fonctions de capitale dans Berlin-Ouest et l'association paradigme patrimonial marxiste-léniniste (comprenant une dimension internationaliste mais aussi une dimension nationaliste) - fonctions de capitale tronquées dans Berlin-Est.

\section{BERLIN RÉUNIfiÉ FACE À DES HÉRITAgES ENCOMBRANTS À PLUSIEURS TITRES : LE NOUVEAU CYCLE PATRIMONIAL DE L'ANCIENNE/NOUVELLE CAPITALE}

La réunification de 1990 et le vote en faveur de Berlin capitale du 20 juin 1991 inaugurent une nouvelle ère dans les relations entre une capitale retrouvée et un patrimoine redéfini. Choisie comme capitale historique - ou head link (Spate, 1942) - de l'Allemagne réunifiée au nom d'un argumentaire patrimonial, Berlin entre dans une phase d'intense recyclage urbain et dans un nouveau cycle patrimonial. Conformément à un schéma classique, le basculement dans l'ancien régime (ici de la RDA) entraîne un tri, avec ses destructions et ses nouveaux patrimoines (le processus de patrimonialisation, par sa dynamique de défonctionnalisation-refonctionnalisation, recycle les héritages), rendu particulièrement crucial par le statut de vitrine de la capitale. Ville-exposition, Berlin est censée être « le lieu qui expose aux yeux du monde 
les valeurs de la société démocratique» (Manale, 2002). Le cas berlinois se trouve toutefois compliqué par l'omniprésence des héritages "polyencombrants" (ou encombrants à plusieurs titres : socialiste, nazi, voire prussien). La ville présente, en effet,

une imbrication particulièrement marquée à Berlin des héritages des différents régimes : de nombreux bâtiments ont été réemployés à différentes époques, si bien qu'il est difficile de savoir de quelle(s) époque(s) et à quel(s) titre(s) ils constituent - ou pas un patrimoine...

\section{A. L'argumentaire patrimonial présidant au retour des fonctions de capitale à Berlin}

Le choix de localisation de la capitale de l'Allemagne réunifiée apparait tout d'abord régi par un argumentaire historique et patrimonial (Djament, Laporte, 2009). Le débat parlementaire aboutissant à un vote favorable, à une courte majorité, au retour du parlement et du gouvernement à Berlin le 20 juin 1991 se révèle structuré par un conflit de légitimité historique et une polémique portant sur l'évaluation de la valeur nationale de l'histoire berlinoise. Revendiquée comme première capitale de l'Allemagne unifiée, Berlin se voit aussi critiquée comme symbole de l'autoritarisme prussien, définitivement discrédité par son statut de capitale du nazisme. Les adversaires de Bonn rétorquent que d'autres villes allemandes, en particulier Munich, ont joué un rôle crucial dans l'histoire du nazisme, et dénoncent le déterminisme consistant à faire de la capitale la cause, et non le siège neutre politiquement, de la politique menée par le régime national-socialiste. L'héritage socialiste de Berlin suscite également la polémique. Tandis que les vestiges matériels et immatériels du régime autoritaire de la RDA sont perçus comme encombrants par les uns, Berlin est associée par les autres au statut d'" avant-poste de la liberté », selon les termes employés par Willy Brandt pour qualifier sa moitié ouest, et à la résistance de sa population lors des épisodes du pont aérien de 1948-49 ou de la révolte populaire de 1953.

Derrière ces débats, la question de fond porte sur l'état de référence privilégié pour la construction de l'Allemagne réunifiée : se positionne-t-elle en tant qu'héritière de la RFA, avec une capitale à Bonn tournée vers l'ouest, ou de l'Allemagne unifiée, avec une capitale à Berlin, interface entre Europe occidentale et Europe centrale qui symbolise la rupture avec la RFA et la fondation d'un nouvel Etat qui retrouve une ancienne nation ? Le maire de Berlin Eberhard Diepgen pose l'alternative en termes particulièrement polémiques : «Il s'agit de la question de savoir si les Allemands veulent l'unification interne des deux parties de leur pays ou seulement une République fédérale allemande agrandie ». Capitale historique controversée, Berlin se trouve finalement désignée comme symbole de coupure surmontée. La difficulté du choix de la capitale révèle toutefois les ambiguités entre réunification et alignement de l'ex-RDA sur la RFA (Carroué, Odent, 1994).

\section{B. Les fonctions politiques de la capitale réunifiée peuvent-elle occuper un patrimoine encombrant?}

Mobilisée dans le choix de localisation de la capitale, la question des héritages fait également débat dans les choix de localisation intra-urbains des fonctions de capitale. 
La nouvelle capitale doit-elle réemployer les bâtiments de l'ancienne capitale ou les détruire?

Le cas du palais de la République est emblématique de la logique de rupture avec l'ancien régime selon laquelle ce qui ne fait plus capitale ne fait pas patrimoine.Après un désamiantage et de longues polémiques, ce haut lieu de la vie politique de la RDA est détruit à partir de février 2006, alors même qu'il faisait patrimoine pour bon nombre d'Ossis pour ses fonctions culturelles et de sociabilité (Hocquet, 2012). Le conflit patrimonial a mobilisé l'Alliance pour le Palais (Palast Bündnis), regroupant des Allemands de l'Est et de l'Ouest partisans du réemploi du Palais, expérimentée sous forme de manifestations et performances artistiques par le festival Volkspalast (Palais $\mathrm{du}$ peuple) puis la Zwischenpalastnützung (réutilisation temporaire du palais à l'abandon)entre 2003 et 2005, et a conduit à une demande de report de la destruction déposée au Bundestag par les Verts et Die Linke (ibid.). Cette "guerre civile des mémoires » (Robin, 2001, p. 200) a débouché sur une forme d'« exclusion symbolique » des Allemands de l'Est (Hocquet, art. cit.). On notera qu'elle reproduit la destruction du bâtiment politique du régime antérieur qui avait déjà été critiquée en 1950 lorsque le régime communiste avait dynamité le château, ne conservant que le balcon d'où Karl Liebknecht avait proclamé, le 9 novembre 1918, la République socialiste.

Le projet de reconstruction du château des Hohenzollern détruit en 1950, voté en 2002 par le Bundestag et un temps bloqué pour des raisons notamment financières ${ }^{12}$, illustre la volonté de reconstruire un patrimoine en éradiquant symboliquement la phase socialiste au profit de l'état antérieur. Il ne manque pas de susciter des débats sur l'évaluation de l'héritage tant socialiste que prussien, mais aussi sur la marchandisation du patrimoine : le projet, lancé par l'homme d'affaires Wilhelm von Boddien, fondateur et secrétaire général de l'association Förderverein Berliner Schloss, et financé par un partenariat public-privé, ést appelé à créer un lieu de consommation (ibid.).

Figure 1 : Prospectus en faveur de la reconstruction du château de Berlin (août 2010)
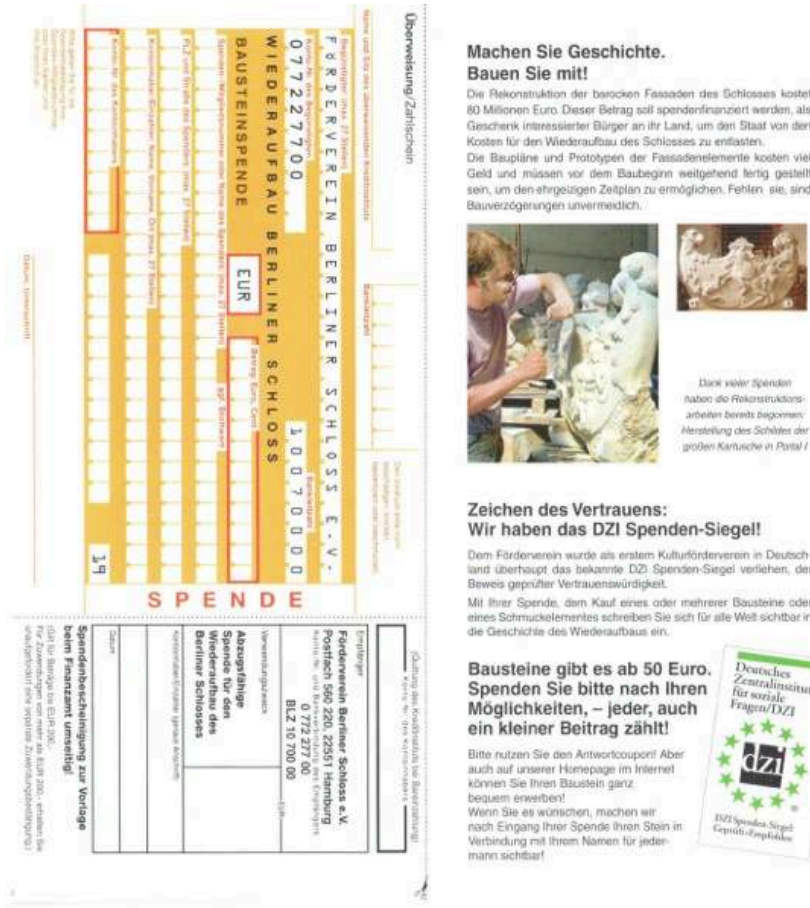

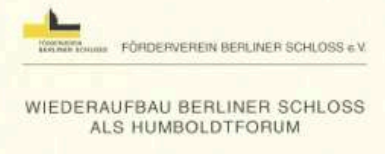

Machen Sie Geschichte! 
Das Schloss lag nicht in Berlin Berlin war das Schloss

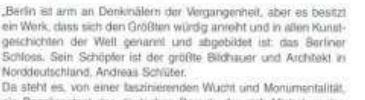

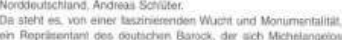
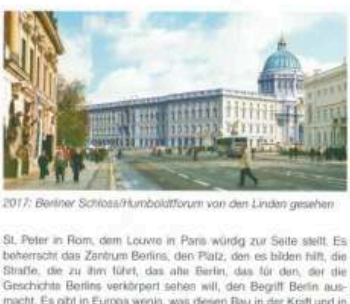

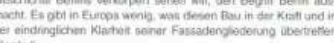

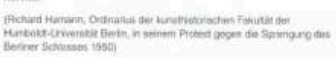

Eine Vision wird Wirklichkeit:

Der Wiederaubau des Schlosses -

Errichtung des Humboldttorums

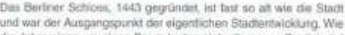

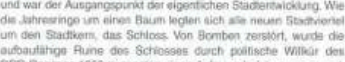

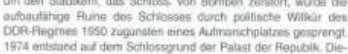

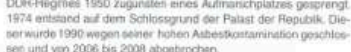

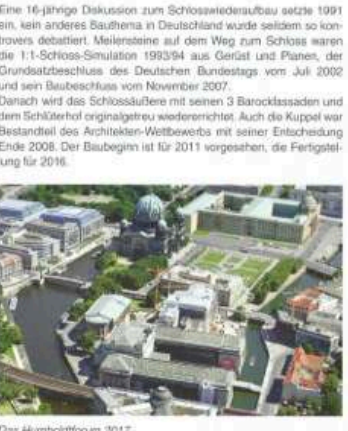

Ein Schioss fûr die Kultur:

Das Humboldtforum

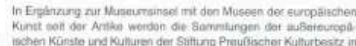

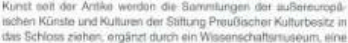

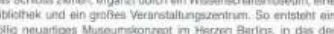

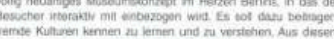

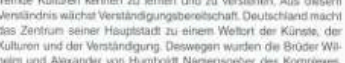

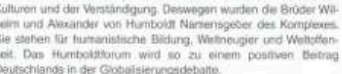

in Bertin bathnt sich nein neves Weltrunder
oter Kanste ants
Förderverein Berliner Schloss e.V.

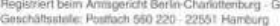

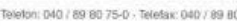

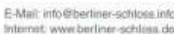

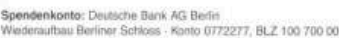

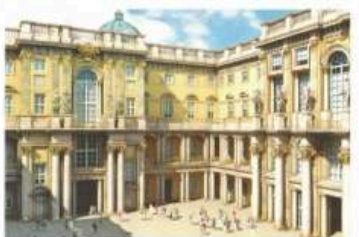

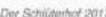

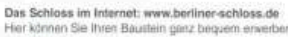

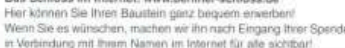

Irmpressum

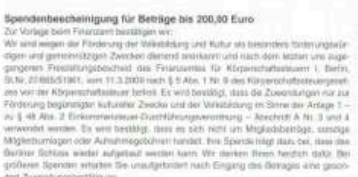

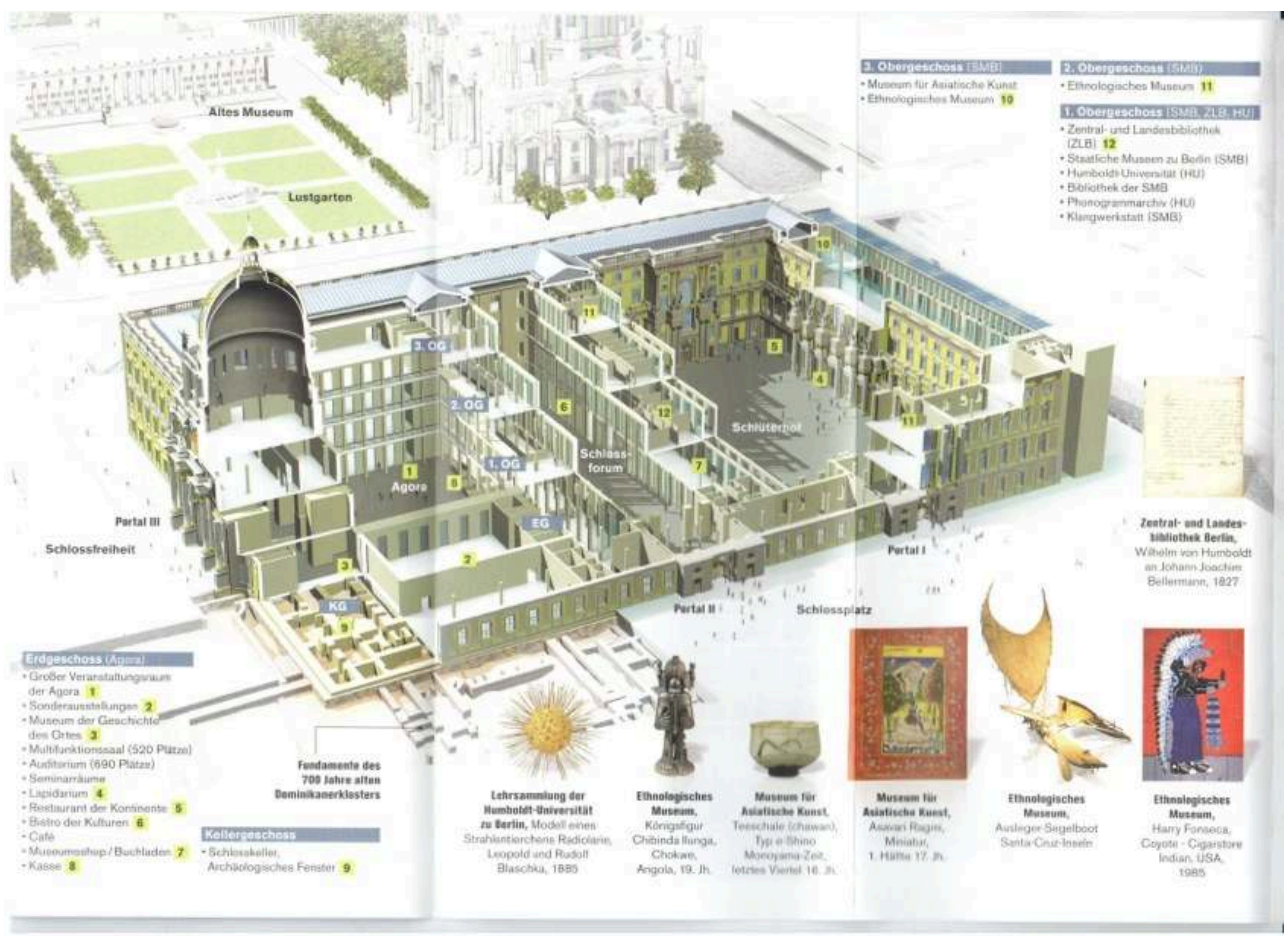

Malgré cette rupture symbolique, le réemploi de bâtiments ayant accueilli la capitale de la RDA ou la capitale de l'Allemagne unifiée préside fréquemment ${ }^{13}$ aux fonctions de capitale réunifiée ${ }^{14}$. Ce choix nécessite de désamorcer la charge "encombrante » des souvenirs et/ou des symboles associés aux bâtiments. L'exemple du réemploi du Reichstag, qui attire aujourd'hui plus de trois millions de visiteurs par an, est emblématique. Lieu de mémoire allemand par excellence, dont le fronton est surmonté de l'inscription «Dem Deutschen Volke $»^{15}$, il est associé à de multiples références historiques (notamment en 1918 la proclamation de la République, l'incendie de 1933, la célèbre photographie représentant la libération par un soldat russe en 1945) et à un 
héritage prussien problématique. Aussi son réemploi a-t-il suscité des polémiques politiques conduisant au rejet du premier projet de N. Foster et à un vote serré en 1994 et s'est accompagné d'un changement de nom officiel ${ }^{16}$ (Ross, 2008). Finalement, l'art contemporain a été utilisé pour désamorcer sa charge encombrante, sur le mode de la performance avec l'emballage de Christo préalable à la rénovation, puis sur le mode de la starchitecture, avec le deuxième projet de $\mathrm{N}$. Foster qui se réfère à l'état d'origine (une coupole moderne) mais le transforme et utilise le verre comme symbole de transparence démocratique, permettant à la République de Berlin de prendre corps et se mettre en scène (Grésillon, Kohler, 2001).

\section{Effacement des traces ou patrimonialisation dans la capitale post-socialiste?}

Par-delà la question éminemment symbolique de l'implantation des fonctions de capitale, l'aménagement de la capitale réunifiée se trouve en permanence confronté à la gestion de traces, de marques, de mémoires jugées encombrantes, souvent à plus d'un titre, et du nouveau marquage ou de la resémantisation de l'espace (Reitel, 2013). Se pose notamment la question des relations entre les différentes "strates " jugées encombrantes, question dont les enjeux font interférer l'évolution du tissu urbain berlinois et la construction de la mémoire allemande : l'héritage prussien doit-il être disqualifié par l'héritage nazi qu'il aurait annoncé ? faut-il mettre sur le même plan l'héritage communiste et l'héritage nazi ?

La logique d'effacement des marques du passé socialiste se révèle bien présente, dans les nombreux changements toponymiques (par exemple la Leninplatz, qui avait remplacé la Landsberger Platz en 1950, devint en 1992 Platz der Vereinten Nationen) ou dans la remise en cause de l'art public socialiste. Ainsi, la statue monumentale de Lénine dans le quartier de Friedrichshain, qui représentait un héritage encombrant à la fois par son emprise spatiale et par sa charge idéologique, est démontée en 1991.

Néanmoins, une patrimonialisation des vestiges socialistes a eu lieu. En témoigne par exemple le Musée de la Stasi-Centre d'études et monument commémoratif de la Normannenstrasse.Fruit de la muséalisation progressivement mise en place sur les lieux mêmes de l'histoire à la suite de l'occupation et de la mise à sac des locaux de la Stasi le 15 janvier 1990 par les manifestants, il commémore la résistance en RDA et la révolution pacifique de 1989-90 (Hocquet, 2011). Cette patrimonialisation se révèle toutefois porteuse de tensions. Projet de musée associatif, initiative citoyenne, le musée de la Stasi s'est trouvé « en conflit avec les logiques des experts mandatés par le Bund pour orienter le travail sur le passé socialiste» (ibid., p. 447). En effet, la logique de «reconnaissance d'un groupe social local (les victimes berlinoises du MfS, porteur d'une mémoire qui se fonde sur une expérience vécue" s'oppose à un projet d'approfondissement de l'institutionnalisation du musée, auquel une portée nationale est conférée, "au sein d'un réseau de "lieux de mémoire et d'apprentissage " susceptibles d'incarner une mémoire culturelle (officielle) de la RDA » (ibid., p. 448).

Dans un tout autre registre, un élément du paysage urbain de RDA comme le feu piéton pour traverser les rues (Ämpelmann) a été défendu contre les projets de changement et s'est même diffusé vers la moitié ouest de la ville. Son érection en "patrimoine » (au sens non institutionnel de symbole identitaire méritant d'être pérennisé et transmis) 
urbain témoigne à la fois d'une certaine "Ostalgie ", mais aussi d'une récupérationmarchandisation comme symbole urbain consommé par les touristes.

Cependant, l'opposition binaire de la destruction et de la patrimonialisation s'avère simpliste au regard de la complexité du rapport que la capitale de l'Allemagne réunifiée entretient à son passé. Ainsi, l'effacement des traces de la RDA ne se réduit pas à des destructions, mais passe par trois modalités: "l'effacement par disparition et destruction, l'effacement par détournement et réécriture de l'histoire et, paradoxalement, l'oblitération par surexposition criminalisée et muséification. » (Combe, Dufrêne, Robin, 2009, p. 8).

De nombreux cas hybrides sont en outre identifiables dans le Berlin post-socialiste. Par exemple, le Mur de Berlin a fait à la fois l'objet d'une destruction et d'un processus de patrimonialisation, marqué par l'ouverture de musées (Musée de Check Point Charlie, East Side Gallery), mais aussi à partir de 2002 par l'aménagement du parcours piéton et cycliste du Mauerweg qui transforme la discontinuité de l'ancien tracé du Mur en espace public (Reitel, art. cit.). Un mémorial a également été réalisé sur la Bernauerstrasse, sur l'ancien no man's land, en 1998.

Tableau 1 : Les différents traitements des héritages encombrants : essai de typologie illustrée de cas berlinois

\begin{tabular}{|l|l|}
\hline Mode de traitement des héritages encombrants & Exemple \\
\hline Destruction & $\begin{array}{l}\text { Démontage de la statue monumentale de Lénine dans le quartier de } \\
\text { Friedrichshain (1991) }\end{array}$ \\
\hline Destruction-reconstruction de l'état antérieur & $\begin{array}{l}\text { Palais de la République-château des Hohenzollern (projet de } \\
\text { reconstruction) }\end{array}$ \\
\hline Destruction globale-muséalisation partielle & Mur-East Side Gallery et Musée de Check Point Charlie \\
\hline Destruction-patrimonialisation & Mur-Mauerweg \\
\hline Délocalisation & Jardin des statues communistes à Moscou et Budapest \\
\hline Réemploi au prix de suppressions & $\begin{array}{l}\text { Epuration de la décoration wilhelmienne du Reichstag par Paul } \\
\text { Baumgarten (terminée en 1961) }\end{array}$ \\
\hline Réemploi au prix d'une inversion symbolique & Neue Wache transformée en mémorial antifasciste par la RDA \\
\hline Réemploi au prix d'ajouts & Coupole du Reichstag par N. Foster (1999) \\
\hline Réemploi par une performance artistique & Emballage du Reichstag par Christo (1995) \\
\hline Patrimonialisation & Musée de la Stasi \\
\hline Monumentalisation & Mémorial aux jưfs assassinés d'Europe (2005) \\
\hline
\end{tabular}

\section{Berlin réunifié : une capitale et un patrimoine néo- national(e), post-national(e) ou mondial(e)?}

Berlin connait une vaste « recomposition mémorielle » liée à la fin de la guerre froide (François, 2001, p. 17). La ville occupe une place importante dans les lieux de mémoire (François, Schultze, 2001) - par opposition aux milieux de mémoire - que les historiens allemands identifient, avec des collègues étrangers, sur le modèle français (Nora, 1984) 
une dizaine d'années après la réunification, mais aussi dans l'identification en cours de lieux de mémoires européens ${ }^{17}$ (François, 2012).

Tableau 2 : Les lieux de mémoire allemands dans Berlin (François, Schultze, 2001 et 2007)

\begin{tabular}{|l|l|l|l|}
\hline $\begin{array}{l}\text { Rubrique de la version } \\
\text { allemande }\end{array}$ & $\begin{array}{l}\text { Lieu de la version } \\
\text { allemande }\end{array}$ & $\begin{array}{l}\text { Rubrique de la } \\
\text { version française }\end{array}$ & $\begin{array}{l}\text { Lieu de la version } \\
\text { française }\end{array}$ \\
\hline Tome 1 : Reich & Der Führerbunker & & \\
\hline Tome 1 : Reich & Der Reichstag & Division et unité & Le Reichstag \\
\hline Tome 1 : Zerrissenheit & Die Mauer & Division et unité & Le Mur \\
\hline Tome 2: Revolution & $\begin{array}{l}\text { Das Brandenburger } \\
\text { Tor }\end{array}$ & Division et unité & $\begin{array}{l}\text { La porte de } \\
\text { Brandenburg }\end{array}$ \\
\hline Tome 2 : Die Moderne & $\begin{array}{l}\text { Der Palast der } \\
\text { Republik }\end{array}$ & Division et unité & $\begin{array}{l}\text { Le Palais de la } \\
\text { République }\end{array}$ \\
\hline Tome 3: Bildung & Die Museumsinsel & & \\
\hline
\end{tabular}

Mais la ville constitue également un lieu de mémoire au sens géographique du terme (Gauchon, 2008), confronté à l'omniprésence des héritages encombrants et la problématique de la réunification non seulement des systèmes patrimoniaux mais surtout des communautés imaginées allemandes. Le choix de l'état (ou des états) de référence du patrimoine réunifié, et, par-delà, de la communauté imaginée allemande, se révèle particulièrement difficile : convient-il de se référer au seul «modèle » ouestallemand? à l'Allemagne d'avant le rideau de fer? d'avant le nazisme? L'hésitation entre un retour au national et un choix post-national effectué sous la RFA, réclamé pour raisons éthiques par certains en Allemagne, se lit dans de nouveaux dispositifs patrimoniaux porteurs d'un nouveau récit historique, tandis que la mondialisation du patrimoine se diffuse.

\section{A. La difficile réunification du patrimoine berlinois}

La réunification du patrimoine pose non seulement des problèmes « techniques ", mais aussi des problèmes de muséographie dans un contexte marqué par la recomposition des identités allemandes. L'Allemagne réunifiée oscille entre la mise en récit d'une identité allemande retrouvée et la mise en récit d'une identité post-nationale et européenne.

Le passage de deux systèmes patrimoniaux à un seul implique tout d'abord une refonte de la " gouvernance " patrimoniale. La moitié est de la ville passe d'un centralisme à un fédéralisme de coopération en matière culturelle, même si l'Etat fédéral intervient 
particulièrement, notamment dans le cadre du fonds de soutien à la capitale (Haupstadtkulturförderung) institué en 1995 puis du « contrat de capitale » conclu pour les années 2001-2004 avec le Land de Berlin (Charléty, 2007). Les grands musées de Berlin-Est sont en 1992 rattachés à la Fondation du patrimoine culturel prussien (Stiftung Preussiger Kulturbesitz), financée par la Fédération et par les Länder, «qui devient la plus importante institution culturelle d'Allemagne financée par le Bund. D'autres musées berlinois sont regroupés dans la Fondation du musée de la ville (Stiftung Stadtmuseum)»(Charléty, 2007, p. 77).

Cependant, la dissymétrie entre une Allemagne de l'Ouest dominante et une Allemagne de l'Est «absorbée » se manifeste notamment dans le Musée historique allemand, temporairement ouvert depuis 1991, définitivement depuis 2006. Remplaçant le projet ouest-allemand évoqué ci-dessus, prévu face au Reichstag à proximité immédiate du Mur $^{18}$, ce musée succède dans l'ancien Arsenal des rois de Prusse au Musée d'histoire allemande de Berlin de RDA (cf. supra). Si la réunification des personnels a eu lieu, la muséographie de l'est est éliminée - une première exposition «Adieux et commencement » se tient en 1990 -, tandis que celle de l'ouest se trouve confrontée à la question du retour du national. L'intervention de l'architecte Pei dans le bâtiment agrandissant le Zeughaus (Arsenal) en 2004, avec son architecture de béton et de verre, son escalier en colimaçon et sa verrière au-dessus de la cour carrée du bâtiment prussien, illustre une recherche de transparence. Si la «réunification » muséale a eu lieu, celle de la communauté imaginée allemande n'a rien d'évident. La mondialisation du patrimoine se manifeste également dans le recours à un "starchitecte».

Quant à l'Ile des Musées, pièce maîtresse du patrimoine berlinois, elle reste incomplètement réunifiée aujourd'hui. Un masterplan visant à sa réunification et à sa restauration est élaboré dès septembre 1990 puis modifié à plusieurs reprises : le projet, qui vise à ramener en centre-ville les collections transférées à Dahlem dans le Berlin de l'après-guerre, a donné et continue à donner lieu à de nombreux débats. En effet, après la Réunification, le directeur général des Musées de Berlin Wolf-Dieter Dube a refusé « d'abandonner la construction du nouveau bâtiment de la Gemäldegalerie de Berlin sur le Kulturforum ${ }^{19}$ qu'il avait planifiée ", considérant que "l'idée d'un retour des peintures sur l'île des Musées - et donc également au Bode-Museum», son lieu d'exposition d'origine, soutenue par l'opinion publique et des critiques d'art, « appartiendrait à « la corbeille à papier de l'Histoire» » (Schuster, 2012). Aujourd'hui, certains militent pour le déplacement de la collection de maîtres anciens du Kulturforum, où elle est peu visitée et où elle serait remplacée par une galerie de peintures du XXe siècle, vers l'Ile des Musées, où une extension du musée Bode serait construite, «de l'autre côté du canal du Kupfergraben, soit juste en face (...), sur ce vaste terrain de casernes sur lequel s'exerçaient autrefois les régiments de la garde du Château de Berlin ", site qui avait déjà été mis à disposition par Helmut Kohl après la Réunification (ibid.). Cette option vient d'être adoptée par la Fondation pour l'Héritage culturel prussien. Elle s'appuie sur une réflexion sur les lieux adéquats pour exposer le patrimoine dans Berlin. Ainsi, l'ancien directeur des musées de Berlin, Peter-Klaus Schuster, dénonce le Kulturforum comme " un emplacement historique erroné », " un lieu qui n'offre pas du tout d'histoire aux Maîtres anciens. Pour eux, c'est un lieu absolument sans histoire, et donc un mauvais lieu. Il en est bien différemment pour l'art du XXe siècle.». Son argumentaire s'appuie à la fois sur l'histoire du lieu, « cœur du Berlin moderne » avec la Neue Nationalgalerie construite par Mies van der Rohe en 1968 et «le bâtiment remarquablement moderne d'Hilmer \& Sattler» pour la 
Gemäldegalerie, et sur le paysage urbain, moderne voire post-moderne, vu du musée : « En tant que paysage urbain pris d'un point de vue historique, le Kulturforum près de la Potsdamer Platz est le lieu le plus inapproprié que l'on puisse imaginer pour les Maitres anciens. Et le public le sent" (ibid.). Il se prononce en faveur de l'Ile des Musées, lieu originel d'exposition des maîtres anciens, au nom de la cohérence de l'ensemble muséal et de l'historicité, et symétriquement pour la transformation du Kulturforum en "Île des Musées de la modernité » (ibid.). Cette réflexion sur l'insertion urbaine des musées plaide pour une logique patrimoniale de localisation du patrimoine. Cependant, d'autres se prononcent pour le déplacement des tableaux anciens de la Gemäldegalerie à proximité du quartier gouvernemental de la nouvelle capitale, dans le château des Hohenzollern à reconstruire où il avait initialement été envisagé de localiser les collections extra-européennes de Dahlem (Museen für Völkerkunde, Ostasiatische und Indische Kunst). L'issue de ces polémiques en cours dépendra entre autres de considérations financières : «La Fondation pour l'Héritage Culturel Prussien annonce qu'après 2018, un nouveau musée de peinture ancienne serait construit près du Bode Museum, sur l'île des Musées » mais le nouveau musée ne dispose actuellement d'aucun financement et la Gemäldegalerie risque de fermer pour des années (Rykner, 2012); la reconstruction du château,suspendue depuis juin 2010 pour cause de crise financière (Aronsson, Bentz, art. cit., p. 341), a débutéen juin 2013.

La question du patrimoine réunifié se trouve ainsi redoublée et compliquée par la notion paradoxale (puisque le patrimoine est transmis en principe du passé) de patrimoine reconstruit, que l'on trouve également à Dresde (Voisin, 2007). Ainsi, la chapelle de la Réconciliation, église néo-gothique de la fin du XIXe s. détruite en 1985 par la RDA, a été reconstruite en matériaux modernes sur son emplacement historique dans l'ancien no man's land du Mur.

Néo-national ou post-national, le patrimoine allemand semble encore se chercher. Une solution est partiellement trouvée dans une européanisation du récit patrimonial, qui permet à la fois de surmonter les difficultés à imag(in)er une communauté allemande et de légitimer le projet géopolitique de reconstituer une puissance métropolitaine continentale.

\section{B. Berlin « capitale du remord » ? La patrimonialisation dans une nation entrée dans l'ère du soupçon}

La difficulté du rapport au national tient non seulement à la réunification mais aussi au rapport à la Seconde Guerre mondiale. Le statut de capitale implique en effet «la concentration à Berlin (...) de mémoriaux portant sur les aspects les plus tragiques et les plus criminels de l'histoire proche", dans le cadre d' "une sorte de partage des tâches " patrimoniales entre un niveau national, où « l'accent (est) mis sur les ruptures, les dimensions tragiques » et au niveau régional et local, où sont valorisées « les valeurs patrimoniales et communautaires » et les héritages « positifs » (François, 2001, p. 18).

Berlin se trouve ainsi aux prises avec la contradiction entre le devoir de mémoire et l'irreprésentabilité de la Shoah, ce qui accélère à la fois la muséalisation de la ville, mise systématiquement en mémoire, et les débats, qui portent sur les lieux de la mémoire au sens géographique du terme et sur le type de mise en exposition adéquats. Berlin se trouve dotée de nouveaux lieux de commémoration et d'exposition, y compris des contre-monuments qui renvoient à la distinction allemande entre Denkmal 
(monument officiel) et Mahnmal (commémorant un passé négatif), et voit se développer les expérimentations artistiques et les "mémoires de proximité » (Robin, op. cit., p. 366). Les polémiques ont ponctué les années 1990 et 2000 . Signalons les plus marquantes.

Le projet du Musée juif inauguré en 2001 selon le concept architectural déconstructionniste 'Between the lines' de Daniel Liebeskind, remonte à l'exposition "Leistung und Schicksal" 20 de 1971 qui conduit à la fondation de la Gesellschaftfürein Jüdisches Museum in Berlin e.V. ${ }^{21}$ et franchit un tournant avec le concours architecturalde 1988. Elle s'inscrit au départ dans le cadre de la concurrence entre la RDA, qui lance le projet de reconstruire la Nouvelle Synagogue, construite entre 1859 et 1866, détruite en 1943, et la RFA, qui présente des expositions sur la culture juive au sein du musée de la ville de Berlin (Aronsson, Bentz, art. cit.).

Quant au projet de Mémorial aux juifs assassinés d'Europe, précédé par le circuit "Topographie des Terreurs" installé sur l'ancien siège de la Gestapo, il remonte également à la période antérieure à la chute du Mur. L'historien Eberhard Jäckel le conçoit comme le pendant allemand du Yad Vashem israelien et convainc en 1988 la journaliste Lea Rosh, qui obtient notamment les soutiens de Willy Brandt, Günter Grass et Christa Wolff, de lancer une mobilisation citoyenne. Le soutien officiel des organisations juives allemande et internationale permet le lancement du projet, qui donne lieu à deux concours architecturaux en 1994/95 puis en 1997, concours finalement remporté par le deuxième projet de champ de stèles de Peter Eisenman. Le mémorial dont la construction a débuté en 2003 a ouvert au public en 2005, pour le soixantième anniversaire de la fin de la Seconde Guerre Mondiale, à proximité de la Porte de Brandebourg et du Tiergarten. Sa localisation au centre de la nouvelle capitale allemande représente une véritable " 'confession to historical responsibility' " (ibid.). Les débats de différents ordres que le mémorial a suscités sont révélateurs de la difficile construction d'une mémoire allemande. Un premier débat a porté sur le lieu de mémoire au sens géographique du terme: les camps nazis et/ou Berlin (plusieurs emplacements intra-urbains ont été proposés). Le projet de mémorial « devint un enjeu électoral en 1998. Eberhard Diepgen, le maire CDU de Berlin, déclara qu'il ne laisserait pas sa ville devenir la « capitale du remord » (Robin, op. cit., p. 389). Un deuxième débat a porté sur la sélection mémorielle proposée ${ }^{22}$ : la commémoration des seules victimes juives des nazis l'a finalement emporté en 1999, à une faible majorité, au Bundestag, conduisant à la décision de construire un mémorial pour les Tsiganes dans Tiergarten ${ }^{23}$ et un mémorial pour les homosexuels. La construction ne fut votée par le Parlement qu'en juin 1999 par un vote assez serré (Aronsson, Bentz, art. cit.).

Après les débats architecturaux, une dernière vive polémique a éclaté en octobre 2003, « quand il apparut que le produit destiné à protéger des graffitis les stèles du mémorial était fabriqué par la firme Degussa, dont l'une des filiales, durant la guerre, fabriquait le zyklon $\mathrm{B}$, utilisé dans les chambres à gaz. (...) Les travaux furent arrêtés près d'un mois, puis reprirent, l'architecte assurant que le produit litigieux était le plus adapté, tandis que la firme Degussa expliquait qu'elle contribuait au fonds d'indemnisation des travailleurs forcés et qu'elle finançait elle-même les chercheurs indépendants autorisés à examiner ses archives. ${ }^{24}$. 


\section{Berlin mise aux normes patrimoniales globales}

Cependant, l'entrée de Berlin dans une nouvelle ère patrimoniale s'explique non seulement par ses nouvelles fonctions de capitale de l'Allemagne réunifiée, mais aussi par ses fonctions métropolitaines. Un nouveau régime touristique globalisé de patrimonialité (Gravari-Barbas, art. cit.) tend en effet à ériger le patrimoine en avantage différenciatif dans la compétition métropolitaine. Dans ce contexte, la patrimonialisation berlinoise tend à changer d'échelle (le lien patrie-patrimoine s'affaiblit au profit d'une "patrimondialisation » (ibid.) et d'une européanisation) et apparaît de plus en plus régie par des standards mondialisés. Métropole incomplète en quête d'une meilleure image internationale, en cours de "worlding" (Cochrane et Passmore, 2001), Berlin cherche à se positionner comme métropole culturelle (Grésillon, 2002) et touristique - elle fait d'ailleurs partie des destinations touristiques émergentes en Europe - en se dotant de plusieurs figures patrimoniales de la globalisation, qui conforment le patrimoine à des standards globaux et qui côtoient des figures paysagères de la globalisation ${ }^{25}$ plus classiques, non patrimoniales et verticalisées, à l'image du nouveau centre d'affaires de la Potsdamer Platz investi par de grandes firmes multinationales. Le label Patrimoine mondial est ainsi obtenu en 1990 par les châteaux et parcs de Potsdam, en 1999 par l'Ile des Musées ${ }^{26}$, en 2008 par les cités du modernisme.

Les musées et les monuments berlinois font également appel à la "starchitecture ", comme l'illustrent notamment les interventions de N. Foster au Reichstag ou de Leoh Ming Pei, auteur de la pyramide du Louvre, au Deutsches Historisches Museum (cf. supra).

Le Berlin post-socialiste se caractérise en outre par l'usage d'un référentiel patrimonial dans l'aménagement de ses espaces publics, devenu «un standard international approprié au niveau local » (Fleury, 2010). Ainsi, la référence - contestée par certains pour son conservatisme - à la Gründerzeit, temps de l'Empire idéalisé, guide la " reconstruction critique ${ }^{27}$ de la zone centrale dans le Planwerk Innenstadt de 1994, présenté comme plan d'unification des deux parties de la ville (Reitel, 2008). Il s'agit non pas de reconstruire à l'identique mais de "respecter et réinterpréter les formes historiques » (Fleury, 2010) afin de faire retrouver à la ville son identité européenne et métropolitaine. On notera que classiquement, la référence à l'histoire constitue en fait une référence à une période antérieure permettant de gommer le passé immédiat : les nouveaux bâtiments, les allées et les rues de la boucle de la Spree adoptent un tracé estouest qui dépasse la division de la guerre froide, mais aussi l'axe nord-sud projeté par les Nazis (ibid.).Dans ce cadre, de nombreuses rues et places monumentales du centre sont requalifiées, à l'image, à partir de 1997 sous l'égide du Land de Berlin, de l'avenue Unter den Linden, dont la promenade centrale se trouve reconstituée dans sa forme d'origine et qui est dotée d'un mobilier urbain «conciliant référence à l'histoire et éléments contemporains » (Fleury, 2010). Le lien entre ce référentiel patrimonial et la fonction de capitale mérite d'être souligné : «Dans la tradition européenne, la rue conserve également sa fonction politique. Dans le cadre du programme Bundeshauptstadt (capitale fédérale), il s'agissait d'une part d'aménager un espace urbain à la hauteur de cette fonction de capitale, avec ses rues et places emblématiques, et d'autre part de rompre plus ou moins explicitement avec l'héritage de la Guerre froide » (ibid.). 


\section{CONCLUSION : BERLIN OBSERVATOIRE DES RECOMPOSITIONS DU LIEN PATRIE-PATRIMOINE- CAPITALE}

rescaling. La métropolisation, qui s'accompagne d'une métropolisation du patrimoine et par le patrimoine, pose ainsi la questionmoine post-national, mis au service des fonctions métropolitaines, directement (fonctions culturelles et touristiques) ou indirectement (fonction de représentation).

La nouvelle capitale politique est devenue le « lieu de mémoire central» de l'Allemagne réunifiée (Reichel, 1998). La réunification patrimoniale et la nouvelle vague de patrimonialisation liées au retour des fonctions de capitale l'ont également érigée en capitale des conflits patrimoniauxallemands, qui portent sur le nazisme et l'holocauste, sur le passé communiste et/ou sur le passé prussien. L'épineuse question de ce qui fait patrimoine dans Berlin et de l'état de référence à retenir nous renvoie aux difficultés d'articuler un champ d'expérience "polyencombrant» à un horizon d'attente allemand. Capitale et métropole «en attente» (Grésillon, Kohler, 2001), Berlin, observatoire d'une complexe redéfinition identitaire, oscille ainsi entre une patrimonialisation néo-nationale et une patrimonialisation post-nationale, entre un patrimoine mis au service des fonctions de capitale et un patrimoine mis au service des fonctions métropolitaines.

L'examen des relations entre patrimoine et capitale à Berlin nous permet ainsi d'aborder sous un nouvel angle les problèmes de la construction nationale allemande, mais aussi plus largement de questionner l'hypothèse de la (partielle) sortie du paradigme national-patrimonial.

A l'heure où l'association patrie-patrimoine (Lamy, 1993) - capitale se trouve remise en cause par la nouvelle étape de mondialisation ${ }^{28}$, le cas de Berlin devient non plus tant la manifestation d'un Sonderweg patrimonial qu'un observatoire de nouveaux rapports du patrimoine à la patrie dans les capitales.

\section{BIBLIOGRAPHY}

Aronsson P., Bentz E., 2011, « National Museums in Germany: Anchoring Competing Communities ", inAronsson P., Elgenius G. (eds), Building National Museums in Europe 1750-2010. Conference proceedings from EuNaMus, European National Museums: Identity Politics, the Uses of the Past and the European Citizen, Bologna 28-30 April 2011, EuNaMus Report No 1.

Althusser L., 1976, « Idéologie et appareils idéologiques d'Etat (Notes pour une recherche) », dans Positions, Paris, Editions sociales, 1976, p. 67-125.

Anderson B., 1996, L'imaginaire national : réflexions sur l'origine et l'essor du nationalisme, Paris, Éditions La Découverte, 212 p. 
Beck U. 2006, Qu'est-ce que le cosmopolitisme?, Paris, Aubier, 378 p.

Bobbio L., 1992, Le politiche dei beni culturali in Europa, Bologne, Il Mulino, 261 p.

Bocquet D., 2010, « Hans Stimmann et l'urbanisme berlinois (1970-2006) : un tournant conservateur de la reconstruction critique? ", Città e Storia, V, 2, p. 467-487.

Brenner N., 2004, New state spaces : urban governance and the rescaling of statehood, Oxford, Oxford University Press, 351 p.

Buffet N. et C., 1999, "Berlin ou l'Allemagne en capitale », Matériaux pour l'histoire de notre temps, $\mathrm{n}^{\circ}$ 55-56. p. 25-31.

Carroué L., 1993, «Berlin réunifiée : une nouvelle métropole à vocation internationale en Europe centrale », Annales de Géographie, nº 570, p. 113-130

Carroué L., Odent B., 1994, Allemagne :état d'alerte?, Paris, L’Harmattan, 220 p.

Charléty V., 2005, Berlin en musée: changements socio-politiques et usages du patrimoine, Bruxelles, Peter Lang, $263 \mathrm{p}$.

Charléty V., 2007, « Politique du patrimoine en Allemagne : un fédéralisme de coopération », Culture et musées, vol. 9, n9, p. 69-98.

Combe S., Dufrêne Th., Robin R., 2009, Berlin, L'effacement des traces : 1989-2009, Paris/Lyon, Musée d'histoire contemporaine, BDIC/Fage Editions, $127 \mathrm{p}$.

Cochrane, A., Passmore, A., 2001, «Building a national capital in an age of globalization :the case of Berlin»,Area, 33(4), p. 341-352.

Djament G., Laporte A., 2009, « Comment Berlin devint capitale de l'Allemagne réunifiée. Eléments pour l'analyse d'un événement territorial », L'Espace Géographique, n 2, p. 146-158.

Dorel-Ferré G., 2011, « Les colonies industrielles catalanes : un patrimoine exceptionnel mais encombrant ", Rives méditerranéennes, n³8, p. 43-56

Fleury A., 2010, «Berlin/Istanbul : la rue au service de l'image des métropoles », Géographie et cultures, $\mathrm{n}^{\circ} 71, \mathrm{p} .111-130$.

François E., 1992, « Naissance d'une nation. Le musée historique allemand de Berlin », Vingtième Siècle, $\mathrm{n}^{\circ} 34$, avril-juin 1992. p. 69-84.

François E., Schulze H., 2001, Deutsche Erinnerungsorte, Munich, Beck, vol. I : 724 p., vol. II : 738 p., vol. III : $784 \mathrm{p}$.

François E., Schulze H., 2007, Mémoires allemandes, Paris, Gallimard, 796 p.

François E., Serrier Th., 2012, Lieux de mémoire européens, Paris, La Documentation

française, Documentation photographique $n^{\circ} 8087,63 \mathrm{p}$.

Gauchon C., 2008, «Entre le temps des historiens et l'espace des géographes, la mémoire?

Approche du paysage mémoriel des Glières ", Actes du Congrès national des sociétés historiques et scientifiques de Besançon, 2004.

Grass G., (édition allemande1995) 2001, Toute une histoire (Ein weites Feld), Paris, Seuil, 722 p.

Gravari-Barbas M., 2013, « Tourisme et patrimoine, le temps des synergies ? », Communication faite dans le cadre du colloque organisé par la Commission Nationale Française pour l'UNESCO « Le patrimoine oui, mais quel patrimoine », UNESCO, 3-4 avril 2012, actes à paraître.

Grésillon B., 2002, Berlin capitale culturelle, Paris, Belin, 351 p. 
Grésillon B., Kohler D., 2001, « Berlin capitale en attente », Hérodote, n¹01, p. 96-121.

Hocquet M., 2011, Mémoire, oubli et imaginaires urbains, Etude de deux hauts-lieux de la mémoire communiste à Berlin-Est : le Palais de la République et le Musée de la Stasi, thèse de doctorat en sociologie et anthropologie politique de l'université de Saint Etienne, 483 p.

Hocquet M., 2012, « Les effets d'exclusion du geste destructeur : le cas du Palais de la République à Berlin ». ethnographiques.org, Numéro 24 - juillet 2012, Ethnographies des pratiques patrimoniales : temporalités, territoires, communautés [en ligne]. (http://www.ethnographiques.org/ 2012/ Hocquet - consulté le 4.12.2012)

Hocquet M., 2013, « La patrimonialisation du centre historique de Berlin, un oubli programmé ? Le réaménagement de la place du château », Espaces et sociétés 2013/1 (n 152-153), p. 67-84.

Lamy Y. (dir.), 1993, dossier « Patrie, patrimoines ", Genèses, vol. 11, n¹1, 174 p.

Laporte A., 2012, « La résilience des espaces du pouvoir et de leur structure : l'exemple de Berlin après la Réunification allemande "; in Djament-Tran G., Reghezza-Zitt M. (coord.), Résiliences urbaines. Les villes face aux catastrophes, Paris, éditions Le Manuscrit, collection Fronts pionniers, $\mathrm{p}$. 103-133.

Le Blanc A., 2010, « La conservation des ruines traumatiques, un marqueur ambigu de l'histoire urbaine », L'Espace géographique, 3/2010, tome 39, p. 253-266.

Manale M., 2002, « Berlin capitale : la ville comme exposition », L'Homme et la société, $n^{\circ} 145$, p. 67-88.

Micoud A., 2005, « Patrimonialisation: redire ce qui nous relie ? Un point de vue sociologique »; in Barrère C. et alii, Réinventer le patrimoine. De la culture à l'économie, Paris, L'Harmattan, p. 81-96.

Nora P. (dir.), 1984, Les lieux de mémoire. I La République, Paris, Gallimard, 674 p.

Poulot D., 2001, Patrimoine et musées : l'institution de la culture, Paris, Hachette, 223 p.

Poulot D., 2006, Une histoire du patrimoine en Occident, XVIIIe-XXIe siècle: du monument aux valeurs, Paris, Presses universitaires de France, 192 p.

Reichel P., 1998, L'Allemagne et sa mémoire, Paris, Odile Jacob, 353 p.

Reitel B., 2008, « Pouvoir politique et production d'espace urbain : histoire des politiques d'aménagement urbain à Berlin ", in Vallat C. (dir.), Pérennité urbaine, ou la ville par-delà ses métamorphoses. Vol 1 - Traces, Paris, L'Harmattan, p. 35-48.

Reitel B., 2013, « La valorisation des traces du Mur de Berlin ou la resémantisation de l'espace urbain », in Djament G., San Marco Ph. (coord.), La métropolisation de la culture et du patrimoine, Paris, éditions Le Manuscrit, collection Fronts pionniers, à paraître.

Ross L. A., "The Reichstag: Quest for the Haupstadtkultur in the new Berlin », Vanderbilt Undergraduate Research Journal, printemps 2008, vol. 4, n¹, 6 p.

Robin R., 2001, Berlin Chantiers, Paris, Stock, 445 p.

Rykner D., 2012, « Graves menaces sur la Gemäldegalerie de Berlin », La Tribune de l'art, mercredi 11 juillet 2012, http://www.latribunedelart.com/graves-menaces-sur-la-gemaoldegalerie-deberlin-article003868.html

Sabrow M., 2009, Erinnerunsorte der DDR, Munich, Beck, 620 p.

Schuster P.-K., 2012, « La corbeille à papier de l'Histoire », La Tribune de l'art, lundi 24 septembre 2012, http://www.latribunedelart.com/la-corbeille-a-papier-de-l-histoire-article003967.html 
Spate O. H. K., 1942, «Factors in the development of capital cities », The Geographical Review, n 3, juillet 1942, p. 622-31.

Stora B., 2007, La guerre des mémoires: la France face à son passé colonial, La Tour d'Aigues, Editions de l'Aube, $107 \mathrm{p}$.

Tavoillot P. -H., 2006, « Querelle des historiens (Historikerstreit) », in Mesure S., Savidan P. (dir.), Le dictionnaire des sciences humaines, Paris, PUF, p. 941-943.

Thiesse A.-M., 1999, La création des identités nationales. Europe XVIIIe-XXe siècle, Paris, Points Seuil, $307 \mathrm{p}$.

Voisin C., 2007, « Le centre, la mémoire, l'identité. Des usages de l'histoire dans la (re)construction du Nouveau marché de Dresde », Espaces et sociétés, 3/2007, n 130, p. 87-101.

Walter F., 2004, Les figures paysagères de la nation, Paris, Editions de l'EHESS, 521 p.

Wahnich S., 2011, « L'impossible patrimoine négatif », Les cahiers Irice, 1/2011, n7, p. 47-62.

François E., Serrier Th., 2012, Lieux de mémoire européens, Paris, La Documentation

française, Documentation photographique $n^{\circ} 8087,63 \mathrm{p}$.

\section{NOTES}

1. Prenant acte de ce qu'il n'existe pas plus de patrimoine en soi que de capitale naturelle, l'approche consiste à étudier la façon dont interfèrent la patrimonialisation et la construction de la capitale, et au service de quel projet géopolitique.

2. Conformément à l'équation "à nouvelle capitale nouveau patrimoine »: forme de construction du champ d'expérience, la patrimonialisation est redéfinie lorsqu'un nouveau régime modifie l'horizon d'attente.

3. Voie spéciale: ce terme désigne classiquement la trajectoire historique spécifique de l'Allemagne conduisant au nazisme. Il est ici utilisé dans un sens extensif, pour souligner les spécificités berlinoises.

4. Reconstruisant une identification nationale après la première période de formation de l'Etat-nation.

5. En 1900 se tient à Dresde le premier congrès allemand pour la conservation des monuments.

6. La Madonne de Stalingrad de Kurt Reuber rappelle ainsi les horreurs de la guerre.

7. Prusse. Essai de bilan.

8. Fondations jumelles opposées.

9. Helmut Kohl est un des rares à parler à l'époque de musée national, la référence à la nation se trouvant pour la majorité discréditée par le nazisme (Aronsson, Bentz, art. cit.).

10. La querelle des historiens éclate en 1986 à la suite de la parution d'un article d'E. Nolte invitant à reconsidérer la place du nazisme dans l'histoire et établissant un parallèle entre nazisme et communisme. Tandis que certains historiens plaidaient pour une réconciliation de l'Allemagne avec son histoire, d'autres dénonçaient «la tentation de neutraliser le passé allemand» (Tavoillot, 2006, p. 941).

11. Questions à l'histoire allemande.

12. Le budget prévu étant de 670 millions d'euros.

13. Même si la Chancellerie ne s'est que provisoirement installée dans l'ancien siège du SED, au profit d'une logique de rupture: la Chancellerie du Spreebogen présente des lignes «ostensiblement perpendiculaires à l'ancien axe du Mur orienté nord-sud et ses fondations (sont) clairement situées à l'ouest » (Laporte, 2012). 
14. Le Bundestag s'est implanté dans le Reichstag, et «a aussi installé des bureaux de députés dans un ancien ministère est-allemand sur Unter den Linden. Hors du centre de la ville, le quartier de Pankow est toujours utilisé par quelques ambassades ", « les ambassades étrangères, désormais en demande de place, ont repris, pour celles qui étaient présentes avant 1939, leurs anciennes localisations » (Laporte, 2012), la Treuhand s'est installée dans l'ancien ministère du Plan de la RDA, ancien bâtiment de l'armée de l'air sous le nazisme (Grass, 1995/2001)...

15. Au peuple allemand.

16. Le Reichstag porte officiellement le nom de „Deutscher Bundestag - Plenarbereich Reichstagsgebaüde“ (Ross, art. cit.).

17. L'histoire berlinoise y intervient, sur le mode positif (1989 avec la chute du Mur fait partie des «Mémoires heureuses ») comme négatif (avec "L’Europe des génocides», et la Seconde guerre mondiale).

18. En 1992, le gouvernement fédéral et le Sénat de Berlin renoncent au projet initial et affectent le terrain au nouvel Office fédéral de la Chancellerie (François, 1992).

19. Situé dans un ancien quartier résidentiel de Berlin-Ouest, à proximité de Tiergarten.

20. Réalisations et destin.

21. Société pour un musée juif à Berlin.

22. La Neue Wache se trouve quant à elle une nouvelle fois réinterprétée en 1993, transformée en mémorial à toutes les victimes de la guerre et de la violence (Aronsson, Bentz, art. cit.).

23. La décision a été prise en 2007 et la construction a débuté en 2008.

24. Georges Marion, «L'Allemagne inaugure à Berlin le Mémorial aux victimes de la Shoah », Le Monde, 10/05/2005.

25. Concept proposé en référence aux « figures paysagères de la nation » (Walter, 2004).

26. Déjà protégée à l'échelle nationale au titre des monuments historiques, au terme d'une reformulation de critères et de périmètre issue de la négociation entre échelle nationale et échelle mondiale. La justification proposée par l'Allemagne pour l'inscription insiste sur trois échelles de référence, nationale, européenne et mondiale : «La Museumsinsel illustre en outre comme le montre l'ordre chronologique de ses musées individuels - l'évolution qu'a connu le musée d'art du début du XIXe siècle au XXe siècle : d'abord lieu central des aspirations à l'éducation de la classe moyenne, puis lieu d'identité nationale, et enfin allié du pouvoir impérial. " «Dans le même temps, la Museumsinsel est un exemple architectural remarquable d'un type de construction qui atteste une étape importante dans le développement de l'histoire de l'humanité. Les différentes conceptions des bâtiments individuels de la Museumsinsel illustrent dans un espace confiné le développement typologique du musée d'art européen ».). Quant à la justification d'inscription retenue par l'Unesco, elle insiste plus sur la valeur universelle de l'ensemble muséal, tont en reconnaissant qu'il « représente l'idée prussienne de l'Etat après les guerres napoléoniennes » : «Critère ii La Museumsinsel de Berlin est un ensemble unique de musées illustrant l'évolution de la conception des musées modernes sur plus d'un siècle. Critère iv Le musée d'art est un phénomène social qui doit ses origines à l'époque des Lumières et son extension universelle à la Révolution française. La Museumsinsel est l'exemple le plus remarquable de ce concept ayant pris forme matérielle dans un cadre urbain central symbolique. » (source : Unesco).

27. Concept proposé par le directeur des services d'urbanisme de Berlin de 1990 à fin 2006 (à l'exception de la période 1996-1999, à la suite d'un revers électoral du SPD) Hans Stimmann. La « reconstruction critique » expérimentée à l'ouest est diffusée à l'est de la ville, doté d'un cadre néo-historique consensuel, évitant les références aux héritages encombrants et vecteur d'une gentrification douce (Bocquet, 2010).

28. Le rescaling (Brenner, 2004) affaiblit le rôle de l'échelle nationale au profit de l'échelle métropolitaine, minorant le lien ville-nation au profit du lien métropole- 
monde, tandis que les dynamiques de «patrimondialisation» (Gravari-Barbas, 2013) distendent le lien patrie-patrimoine.

\section{ABSTRACTS}

Inside the nation making process, heritage and capital cities have dialectic relationships, made difficult in Berlin by the regular change of political status and by the peasant heritage ofNazismthat discredited German nationalism.

First of all, the article presents the forming link between heritage and nation in Berlin and the disruptive factors (nazism and Cold War). Then, it analyzes the new heritage cycle created by the status of capital city of reunited Germany and the hesitations about socialist, nazi and prussian heritages (reuse, destruction, heritagization, rebuilding). The numerous memory wars in Berlin show a fluctuation between a neonational and a postnational idea of the capital city and of its heritage. At the same time, heritagization interferes more and more with the metropolitan functions of the city that is seeking to position itself on the international scene. Berlin is a good place for examining the new scales of cities and heritage.

$\mathrm{Au}$ service du processus de construction nationale, la patrimonialisation et les capitales entretiennent un rapport dialectique, complexifié à Berlin par de fréquents changements de statut politique, mais surtout par l'héritage encombrant du nazisme qui a jeté le discrédit sur le nationalisme allemand.

Après avoir rappelé la mise en place du paradigme national-patrimonial dans Berlin et ses perturbations lors du nazisme et de la guerre froide, cet article présente le nouveau cycle patrimonial lié au statut de capitale de l'Allemagne réunifiée et ses hésitations (réemploi, destruction, patrimonialisation, reconstruction) face aux héritages socialistes, nazis et prussiens. Les guerres de mémoire qui se sont multipliées dans Berlin renvoient à une tension entre une conception néo-nationale et une conception post-nationale de la capitale et de son patrimoine. Parallèlement, la patrimonialisation interfère de façon croissante avec les fonctions métropolitaines de la ville qui se repositionne sur la scène internationale. Berlin constitue ainsi un observatoire privilégié des recompositions scalaires liées de l'urbain et du patrimoine.

\section{INDEX}

Schlüsselwörter: Erbe, Erinerrungsorte, Haupstadt, lästiges Erbe, Wende

Mots-clés: capitale, héritages encombrants, lieux de mémoire, patrimonialisation, réunification allemande

Keywords: capital city, german reunification, heritagization, peasant heritages, places of remembrance

\section{AUTHOR}

\section{GÉRALDINE DJAMENT-TRAN}

Géographe - Maître de conférences - Université de Strasbourg 